\title{
Early medical rehabilitation after neurosurgical treatment of malignant brain tumours in Slovenia
}

\author{
Natasa Kos ${ }^{1}$, Boris Kos², Mitja Benedicic ${ }^{3}$ \\ ${ }^{1}$ Medical Rehabilitation Unit, University Medical Centre, Ljubljana, Slovenia \\ ${ }^{2}$ Zdravstveni dom dr. Julija Polca Kamnik, Slovenia \\ ${ }^{3}$ Department of Neurosurgery, University Medical Centre, Ljubljana, Slovenia
}

Radiol Oncol 2016; 50(2): 139-144.

Received 23 September 2014

Accepted 3 December 2014

Correspondence to: Mitja Benedičič, M.D., Ph.D., Department of Neurosurgery, University Medical Centre Ljubljana, Zaloška 2, SI-1000 Ljubljana, Slovenia. E-mail: mitja.benedicic@kclj.si

Disclosure: No potential conflicts of interest were disclosed.

Background. The number of patients with malignant brain tumours is on the rise, but due to the novel treatment methods the survival rates are higher. Despite increased survival the consequences of tumour properties and treatment can have a significant negative effect on the patients' quality of life. Providing timely and appropriate rehabilitation interventions is an important aspect of patient treatment and should be started immediately after surgery. The most important goal of rehabilitation is to prevent complications that could have a negative effect on the patients' ability to function.

Conclusions. By using individually tailored early rehabilitation it is often possible to achieve the patients' independence in mobility as well as in performing daily tasks before leaving the hospital. A more precise evaluation of the patients' functional state after completing additional oncologic therapy should be performed to stratify the patients who should be directed to complex rehabilitation treatment. The chances of a good functional outcome in patients with malignant brain tumours could be increased with good early medical rehabilitation treatment.

Key words: malignant brain tumour; surgery; early rehabilitation

\section{Introduction}

Malignant brain tumours have a very high likelihood of producing disabling effects on a patient's life. The indirect effects of chemotherapy and radiation therapy add to the functional deficits which are usually caused by the tumour itself (mass effect). Neurological deficits are related to the area of the brain that the tumour invades. The most common neurological complications of primary brain tumours are cognitive deficits $(80 \%)$, motor deficits (78\%), visual-perceptual deficits (53\%), sensory loss (38\%), bowel/bladder impairment $(37 \%)$, cranial nerve palsy $(29 \%)$, dysarthria $(27 \%)$, dysphagia (26\%), aphasia (24\%) and ataxia (20\%). ${ }^{1}$ The observed preoperative and postoperative neu- rological deficits have an important impact on patients' daily life functions and result in diminished ability to perform usual family and social roles. Furthermore, most of the patients experience progressive neurological decline as their disease progress. $^{2}$

Brain tumours occur over the life span with higher incidence in advanced age. ${ }^{3}$ The survival rates have increased due to early diagnostics and up-to-date multidisciplinary treatment involving neurologists, neuroradiologists, neurosurgeons, oncologists and the medical rehabilitation specialists. ${ }^{4,5}$ However, besides prolonged survival ${ }^{6}$, the contemporary approach is the maintained or improved quality of life, which can be much contributed to by the rehabilitation processes, which 
needs to be adjusted to the individual's abilities. The purpose of rehabilitation is restoring independence with the emphasis on activities of daily living, mobility, cognition and communication. Interventions can be applied in all stages of the disease but the rehabilitation goals adjust according to the patient's problems. The rehabilitations needs to start early enough in order to reach the established goals, prevent the complications and achieve better functional outcome. ${ }^{7}$

\section{Symptoms of brain tumours}

The symptoms of brain tumours are dependent on the size and location of the tumour. They are caused by mass effect of the tumour and by the surrounding vasogenic brain oedema. First symptom is usually headache, which is usually worse in the morning and can be accompanied by nausea and vomiting. ${ }^{8}$ Sometimes an epileptic seizure is the first and only symptom. Each person may experience symptoms differently, but motor deficits and speech disturbances are the most unpleasant because they interfere with patient's independence. Cognitive deficits are common and important because they have effect on quality of life and on the efficiency of specific rehabilitation programme, but can go unnoticed for longer periods of time., 9,10

\section{Operative treatment of brain tumours}

Surgery is usually the first choice of treatment; the goal of surgery is maximal tumour resection, but it is also important to provide the diagnosis and prevent symptoms of the mass effect. ${ }^{11}$ In addition to microsurgery, several new techniques are used in brain tumour surgery, such as frameless, imageguided neuronavigation, preoperative functional MRI, fiber tracking and transcranial magnetic stimulation, intraoperative ultrasound and MRI, intraoperative neurophysiological monitoring (including direct cortical stimulation), fluorescenceguided removal of malignant gliomas, stereotactic needle biopsy, neuroendoscopy, awake surgery and brachytherapy. These novel techniques can help the surgeon to facilitate tumour removal, minimize the injury of the surrounding brain tissue and the occurrence of postoperative neurological deficit, thus resulting in better patient outocome. ${ }^{11}$

\section{Postoperative period}

The most common complaint after surgery is fatigue, which improves over time, but can be intense during first weeks after surgery and also during chemotherapy or radiation therapy. ${ }^{12}$ Relatively little research on fatigue in patients undergoing surgery for malignant brain tumours has been performed to date. ${ }^{13}$ More severe fatigue significantly correlate with poor functional status and poorer quality of life due to impaired physical functioning and sleep disturbances. ${ }^{13}$ Patients with fatigue have problems with routine tasks - these tasks require greater concentration and effort as usually. These problems need to be considered when deciding upon the intensity of activity during the rehabilitation program. Therefore, we plan short periods of rest during the program and assure that the patients stop with the activity before becoming overtired.

Besides fatigue, cognitive functions such as attention, concentration and memory can be affected to a varying degree. The deficits may be temporary or more permanent, depending on whether the cause is permanent and structural or transient due to temporary brain swelling. Affective disorders must be considered immediately after surgery they are more common in patients with the history of depression and those with coincidental physical disability. ${ }^{14}$ It is important to determine the severity of the cognitive impairments and to accordingly modify the rehabilitation planning. ${ }^{8}$

Headache can last for a few days after the operation and can interfere with the patients' ability to participate in activities. Neurological deficits (paralysis, weakness and balance disturbances) may also persist after the surgery.

\section{Early medical rehabilitation in Slovenia}

Recovery time after surgery is different for each individual. The goal of postoperative rehabilitation is to prevent complications and to maximize the patient's functional abilities. Early rehabilitation at our institution is provided by the rehabilitation team, consisting of the occupational therapist working together with the physiotherapist, while the consultant of physical and rehabilitation medicine is responsible for proper rehabilitation procedures used to help the patient return to normal activities. The rehabilitation procedures are started as soon as possible because the length of hospital stay is short (usually around 1 week) and time to achieve the goal is limited. The content, intensity and frequency of the rehabilitation programme are tailored to the individual patient's clinical needs. Patient and his relatives are also included in the rehabilitation team, while consultants of other specialisations are included as needed. 
On the first postoperative day, provided that the control CT scan of the brain shows no significant postoperative hematoma or oedema, we start with progressive mobilisation. This is possible when the patient has good physical stamina and is without motor deficits. We evaluate the patient's independence in basic daily activities and Karnofsky performance scale (KPS) is used for evaluation of patient's functional abilities. This scale allows patients to be classified as to their functional impairment. The Karnofsky score runs from 100 to 0 , where 100 is "perfect" health and 0 is death - the lower the score, the bigger the impairment. ${ }^{15} \mathrm{KPS}$ may be used to determine patient's prognosis or to measure changes in a patient's ability to function and is often used with patients suffering from malignant brain tumours. ${ }^{16,17}$

Often the patients present with cognitive deficits despite the lack of motor impairment. They would benefit from early neuropsychological treatment or at least early neuropsychological evaluation. However, this can rarely be achieved in the acute phase due to lack of clinical psychologists in hospital setting in Slovenia. Neuropsychological assessment helps to determine whether treatment, in the form of cognitive rehabilitation or psychotherapy, may be useful after discharge from the acute hospital. Outpatient programs to address cognitive deficits in brain tumour survivors, including cognitive therapy and pharmacologic strategies, have shown to be been beneficial. ${ }^{9}$

Patients who experience temporary or permanent speech difficulties require specialised therapy by the speech therapist. The speech therapist is invited into the rehabilitation team early after the operation if speech and swallowing difficulties are detected, but sufficient patient's cooperation must be assured. ${ }^{18}$

Preliminary results of our retrospective review focusing on neurological deficits after surgery for malignant brain tumours during the past three years show that the proportion of patients with persistent neurological deficits is substantial even after surgery and in about $30 \%$ percent of patients neurological deficits could be identified at discharge. Neurological deficits can often be multiple, Mukand et al. described that $74.5 \%$ of patients had three or more concurrent neurologic deficits, and $39 \%$ of patients had five or more deficits, which is in accordance to our preliminary results. ${ }^{1}$

Patient with neurological deficits need special rehabilitation while they are in the acute hospital. Usually, the most important deficit for patients is motor impairment and problems with walking.
Such a patient is bed-ridden and the resulting complications need to be prevented. In this setting, verticalization is very important and there are usually no contraindications for it. We use different equipment to help patients sit on their bed, the tilt table is used to achieve standing and the wheelchairs are used for sitting. With the wheelchair, we enable the patient to be driven out from the room. This has a positive effect on the patient's wellbeing, preventing social isolation which may occur when the patient is constantly in bed and alone in the room.

With the help of a chosen neuro-physiotherapeutic technique we opt to maintain a good passive range of movement in the joints of the limbs where active movement is not possible. When increased muscle tone is present, these techniques also contribute to its normalization and help the patient to start using paretic limbs. Occupational therapist evaluates and treats difficulties, related to selfcare and daily living and plays an important role in helping the patient develop new ways of doing different daily tasks, such as dressing, undressing, washing and eating. Often, while in the acute hospital, the patient is provided with individually tailored accessories to facilitate functioning at home (thickened cutlery handles, equipment to assist with putting on footwear etc.). Additional instructions are provided to the patient and their relatives in order to improve the organization of daily living at home.

Rehabilitation does not end with the patient's discharge from the acute hospital. Hospital stays are short; at our institution, the average length of stay in the acute hospital is 9 days, which often means that the rehabilitations goals are not fully met. The patients are either discharged home or transferred to other hospitals and most of them must continue with additional oncologic treatment.

University Rehabilitation Institute of the Republic of Slovenia is the only tertiary institution performing complex rehabilitation in Slovenia. Direct transferral of patient with malignant brain tumours from the acute hospital to this institution are rare, since most patients need further oncologic treatment; transferrals are thus only possible after the oncologic treatment is complete.

Some patients remain very weak, dependant and immobile due to the extent of neurological and cognitive impairment. When it comes to extensive neurological deficits and disturbances of consciousness with poor cooperation, our actions are mainly focused on preventing complications arising from constantly lying in bed. Special emphasis is put on respiratory physiotherapy. It is 
necessary to regularly turn the patient, put him in proper positions and perform neuro-physiotherapy; electronic devices that enable joint movements and prevent contractures can also be used. Even patients in the minimally conscious state can use the wheelchair, provided it has been adapted to accommodate for passive sitting. Patients in the minimally conscious state can be stimulated with different sensory stimuli in the appropriate environmental settings, minimising additional disruptive stimuli from the surroundings. Relatives help in picking the appropriate stimuli and can also perform sensory stimulation during visiting hours. It is extremely important to educate the family to be able to offer good care and support to the patient, especially when patients are bedridden and dependant in daily activities needing the help of other people. Often, it is also necessary to arrange for proper utilities the patient will need at home, such as an adopted bed, wheelchair, walker or lift for easier handling with bedridden patients. Such equipment can be prescribed when the patient is discharged from the hospital.

\section{Discussion}

Increasing incidence of brain tumours has been observed in many countries over the last thirty years. ${ }^{19}$ In assessing the outcome of malignant brain tumour patients, lift expectancy as well as direct and indirect functional impairment must also be taken into consideration. Giordana et al. have reviewed several studies dealing with the functional outcome of brain tumour patients and have shown that rehabilitation intervention offers significant benefit to this patients. ${ }^{20}$ The rehabilitation process is therefore of paramount importance in brain tumour patients when compared to other malignancies because of their extremely high rate of associated disability. ${ }^{3}$ Similarly, preliminary results of our retrospective review have shown that approximately $30 \%$ of 200 patients being treated annually for malignant brain tumours have postoperative neurological deficits. Patients usually receive further oncologic treatment and we can assume that their functional status might worsen during additional therapy due to brain oedema or tumour progression. Recent progress in the multimodal treatment of brain tumour patients has improved 5 year survival rate, which has resulted in an increased number of patients requiring rehabilitation support. ${ }^{3}$ Nowadays cancer is viewed as a chronic disease where rehabilitation becomes an important aspect of care. However, despite the high incidence of neurological and functional deficits in brain tumour patients, rehabilitation treatment in this population is not as well established as it is for patients with other neurological conditions. ${ }^{7}$ In a study comparing brain tumour patients and patients with traumatic brain injury the authors found no significant differences in mobility and independence in activities of daily living between both groups of patients. ${ }^{21}$ Functional improvements are also comparable to those achieved in stroke patients. ${ }^{20}$ Clinical guidelines suggest that rehabilitation should begin early in stroke patients in order to improve the recovery process and reduce disability. ${ }^{22,23}$ In brain tumour patients, where deterioration is often faster than in stroke patients, the need for early intervention is even more pressing. ${ }^{7}$ Rehabilitation, especially during the acute phase and immediately postoperatively can improve functional outcome. ${ }^{7}$ The review of the literature by Khan et al. failed to identify and high quality studies evaluating the effectiveness of multidisciplinary rehabilitation care in patients with brain tumours. ${ }^{24}$ On the other hand, there is strong evidence that unidisciplinary interventions (exercise, physical therapy...) enhance functional outcome and improve quality of life. ${ }^{24}$

Rehabilitation is recommended in early stages of the disease for function restoration after surgery and in more advanced stages as an important part of palliative care with the aim to prevent complications, control the symptoms and maintain patients' independence and quality of life regardless of life expectancy. ${ }^{25}$ In brain tumour patients, specificity of medical treatment, complication of surgery and side effects of irradiation and chemotherapy have to be taken into consideration. ${ }^{26}$ Side effects of corticosteroids and anticonvulsants are also important, because their chronic use can be associated with myopathy, osteoporosis, behavioural changed and psychiatric disorders. ${ }^{27}$ Anticonvulsants can affect cognitive functions, alter the reaction time and in some instances cause movement disorders such as ataxia; all of these side effects influence the rehabilitation process. ${ }^{26}$ In the acute phase of rehabilitation, flexibility and frequent reassessments are required. ${ }^{28}$ Oncologic and other treatments may impact the timing of physical therapy interventions, which should be performed in a phase of patient's peak performance. ${ }^{28}$ Because of diverse clinical picture and varying levels of disability among brain tumour patients an individualised approach is always warranted. ${ }^{29}$ 
With proper early medical rehabilitation in the postoperative settings we enable the patients to become more independent, prevent complications and increase baseline conditions for further rehabilitation. The length of stay in the acute hospital setting is short and we start with rehabilitation procedures immediately after surgery; these procedures should be carried out throughout the additional oncologic therapy. After finishing the treatment, the patients should be evaluated and the neurological impairments, influencing daily functioning, should be identified. Further complex rehabilitation could be arranged at the University Rehabilitation Institute of the Republic of Slovenia. Several studies have shown significant improvement in the functional state of brain tumour patients as a result of in inpatient rehabilitation. ${ }^{30,31}$ In the institutions providing inpatient rehabilitation, patients are offered individually adapted treatment programs based on their deficit, ability to cooperate and set goals. The challenge is not just to help the patients overcome their disabilities and improve performance status but also to help them stay independent in the community and lower the family burden. After finishing the inpatient rehabilitation, the patients may also receive appropriate rehabilitation devices to facilitate care giving at home and prolong their independency.

We must emphasize that an article by Goljar from 2008 shows that only about 10 patients treated for malignant brain tumours are admitted to the University Rehabilitation Institute of the Republic of Slovenia yearly. ${ }^{32}$ Considering large number of patients undergoing surgery and a significant proportion of postoperative neurological symptoms it remains unclear what type of rehabilitation is offered to them after completion of the oncologic treatment.

Besides motor impairments, an important potential long-term deficit of the surviving patients is cognitive deterioration, which is related to tumour location, surgical morbidity and oncologic treatment. ${ }^{9}$ Problems related to cognitive impairment are well documented and present even in the tumour remission period. ${ }^{33}$ However, only few studies have focused on strategies to prevent and treat cognitive deficits in brain tumour patients and many of them have serious methodological limitations, such as too small of a sample or retrospective study design, so further studies are necessary. ${ }^{33,34}$ Regardless of the severity, cognitive deficits have a significant negative impact on patient's daily living. ${ }^{35}$ In 2003, Hahn and as- sociates published a prospective study, in which they performed standardised neuropsychological testing and life quality evaluation in primary brain tumour patients. ${ }^{36}$ The study has shown that individually tailored rehabilitation programs can increase the patient's quality of life. ${ }^{36}$ Gehring et al. have in 2008 established that we are at a relatively early stage of development of effective pharmacological and behavioural approaches towards treatment and prevention of cognitive deficits in this group of patients, although the approaches used might have a positive effect on other areas of patient's functioning and well-being. ${ }^{33}$ Cognitive deficits interfere significantly with familial, social and career-related activities. ${ }^{37}$ Recent systematic review of the literature established that neurocognitive symptoms and personality changes irreversibly altered the relationship between the patient and the caregiver, giving the caregiver the sense of total responsibility. ${ }^{38}$ For the patient, however, loss of autonomy and coping with restriction seem to be the most difficult. ${ }^{38}$ Early identification of neuropsychological changes may lead to improved effectiveness of cognitive training. ${ }^{33}$ It has been shown that early cognitive training in the postsurgical period markedly improves cognitive functioning. ${ }^{33}$ Taking into account and treating these impairments can enhance the patients' quality of life. Therefore, more precise evaluation of patients' functional and cognitive state after completion of the oncologic therapy is warranted to establish criteria for further complex medical rehabilitation.

\section{Conclusions}

Rehabilitation of patients suffering from malignant brain tumours is an important part of treatment. By starting treatment early during hospitalization and by continuing the treatment in qualified institutions later on we can raise the quality of the patient's life and achieve higher levels of independence. Considering the high number of patients in Slovenia the percentage of those referred to the University Rehabilitation Institute of Slovenia after oncologic treatment is low. We believe that a more precise evaluation of the patients' functional state after finishing additional tumour related treatments should be performed and more patients directed to the complex rehabilitation treatment. In addition to retrospective reviews, a well-designed study should be performed in Slovenia to establish the effect of inpatient postoperative rehabilitation on the quality of life in this group of patients. 


\section{References}

1. Mukand JA, Blackinton DD, Cirncoli MG, Lee JJ, Santos BB. Incidence of neurologic deficits and rehabilitation of patients with brain tumors. Am J Phys Med Rehabil 2001; 80: 346-50.

2. Hansen A, Rosenbek Minet LK, Sogaard K, Jarden JO. The effect of an interdisciplinary rehabilitation intervation comparing HRQoL, symptom burden and physical function among patients with primary glioma: an RTC study protocol. BMJ Open 2014; 4(10): e005490.

3. Vargo M. Brain tumor rehabilitation. Am J Phys Med Rehabil 2011; 90(5 Suppl 1): S50-62.

4. Kase M, Minajeva A, Niinepuu K, Kase S, Vardja M, Asser T, et al. Impact of $\mathrm{CD} 133$ positive stem cell proportion on survival in patients with glioblastoma multiforme. Radiol Oncol 2013; 47: 405-10.

5. Maza S, Buchert R, Brenner W, Munz DL, Thiel E, Korfel A, et al. Brain and whole-body FDG-PET in diagnosis, treatment monitoring and long-term follow-up of primary CNS lymphoma. Radiol Oncol 2013; 47: 103-10.

6. Smrdel U, Kovac V, Popovic M, Zwitter M. Glioblastoma patients in Slovenia from 1997 to 2008. Radiol Oncol 2014; 48: 72-9.

7. Bartolo M, Zucchella C, Pace A, Lanzetta G, Vecchione C, Bartolo M, Grillea G, Serrao M, Tassorell C, Sandrini G, Pierelli F. Early rehabilitation after surgery improves functional outcome in inpatients with brain tumours. $J$ Neurooncol 2012; 107: 537-44.

8. Cheville AL. Cancer rehabilitation. In: Braddon LB, Chan L, Harrast MA, et al, editors. Physical medicine and rehabilitation. $4^{\text {th }}$ edition, Philadelphia: Saunders 2011. p. 1371-401.

9. Shen C, Bao WM, Yang BJ, Xie R, Cao XY, et al. Cognitive deficits in patients with brain tumor. Chin Med J 2012; 125: 2610-7.

10. Giovagnoli AR. Investigation of cognitive impairments in people with brain tumors. J Neurooncol 2012; 108: 277-83.

11. Vranic A. New developments in surgery of malignant gliomas. Radiol Oncol 2011; 45: 159-65.

12. Tuna-Malak A, Diramali A. Radiotherapy-related tiredness in patients with glioblastoma multiforme (GBM). Asian Pac J Cancer Prev 2008; 9: 497-500.

13. Kim RB, Chun MH, Han EY. Fatigue assessment and rehabilitation outcomes in patients with brain tumors. Support Care Cancer 2012; 20: 805-12.

14. Mainio A, Hakko H, Niemela A, Koivukangas J, Rasanen P. Depression and functional outcome in patients with brain tumours: a population-based 1-year follow-up study. J Neurosurg 2005; 103: 841-7.

15. Vincent Mor V, Laliberte L, Morris JN, Wiemann M. The Karnofsky performance status scale: An examination of its reliability and validity in a research setting. Cancer 1984; 53: 2002-7.

16. Schag CC, Heinrich RL, Ganz PA. Karnofsky performance status revisited Reliability, validity, and guidelines. J Clin Oncol 1984; 2: 187-93

17. O'Toole DM, Golden AM. Evaluating cancer patients for rehabilitation potential. West J Med 1991; 155: 384-7.

18. Kos N. [Early medical rehabilitation after surger of central nervous system tumors]. [Slovenian]. In: Marinček Č, Burger $\mathrm{H}$, editors. $19^{\text {th }}$ rehabilitation medicine days, Ljubljana, March 28-29, 2008. Rehabilitacija 2008; 7(Suppl 2): 49-51.

19. Wrensch M, Minn Y, Chew T, Bondy M, Berger MS. Epidemiology of primary brain tumors: Current concepts and review of the literature. Neuro Oncol 2002; 4: 278-99.

20. Giordana MT, Clara E. Functional rehabilitation and brain tumour patients. A review of outcome. Neurol Sci 2006; 27: 240-4.

21. Huang ME, Cifu DX, Keyser-Marcus L. Functional outcomes in patients with brain tumor after inpatient rehabilitation: comparison of traumatic brain injury. Am J Phys Med Rehabil 2000; 79: 327-35.

22. Duncan PW, Zorowitz R, Bates B, Choi JY, Glasberg JJ, Graham GD, Katz RC, Lamberty K, Reker D. Management of adult stroke rehabilitation care: a clinical practice guideline. Stroke 2005; 36: 2100-43.

23. Bates B, Choi JY, Duncan PW, Glasberg JJ, Graham GD, Katz RC, Lamerty K, Reker D, Zorowitz R. Veterans Affairs/Department of Defense Clinical Practise Guideline for the Management of Adult Stroke rehabilitation care: executive summary. Stroke 2005; 36: 2049-56.
24. Khan F, Amatya B, Ng L, Drummond K, Olver J. Multidisciplinary rehabilitation after primary brain tumour treatment (review). Top Geriatr Rehabil 2011; 27: 184-92.

25. Bartolo M, Zucchella C, Pace A, De Nunzio AM, Serrao M, Sandrini G, et al. Improving neuro-oncological patients care: basic and practical concepts for nurse specialist in neuro-rehabilitation. J Exp Clin Cancer Res 2012; 31: 82.

26. Ching W, Luhmann M. Neuro-oncologic physical therapy for older person. Top Geriatr Rehabil 2011; 27: 184-92.

27. Pace A, Metro G, Fabi A. Supportive care in neurooncology. Curr Opin Oncol 2010; 22: 621-6.

28. Kirshblum S, O'Dell MW, Ho C, Barr K. Rehabilitation of persons with central nervous system tumors. Cancer 2001; 92(4 Suppl): 1029-38.

29. Sherwood PR, Given BA, Given CW, Schiffman RF, Murman DL, Lovely M, et al. Predictors of distress in caregivers of persons with a primary malignant brain tumor. Res Nursing Health 2006; 29: 105-20.

30. O'Dell MW. Barr K, Spanier D, Warnick RE. Functional outcome of inpatient rehabilitation in persons with brain tumors. Arch Phys Med Rehabil 1998; 97: $1530-4$

31. Marciniak CM, Sliwia JA, Heinemann AW, Semik PE. Functional outcomes of persons with brain tumors after inpatients rehabilitation. Arch Phys Med Rehabil 2001; 82: 457-63.

32. Goljar N. [Comprehensive rehabilitation of patients with brain tumours] [Slovenian]. In: Marinček Č, Burger $\mathrm{H}$, editors. $19^{\text {th }}$ rehabilitation medicine days, Ljubljana, March 28-29, 2008. Rehabilitacija 2008; 7(Suppl 2): 52-5.

33. Gehring K, Sitskoom MM, Aaronson N, Tophoom MJB. Interventions for cognitive deficits in adult with brain tumours. Lancet Neurol 2008; 7: 548-60.

34. Weitzner MA, Meyers CA. Cognitive functioning and quality of life in malignant glioma patients: A review of the literature. Psychooncology 1997; 6: 169-77.

35. Zucchella C, Capone A, Codella V, De Nunzio AM, Vecchione C, Sandrini G, et al. Cognitive rehabilitation for early post-surgery inpatients affected by primary brain tumor: a randomized, controlled trial. J Neurooncol 2013; 114: $93-100$.

36. Hahn CA, Dunn RH, Logue PE, King JH, Edwards CL, Halperin EC. Prospective study of neuropsychologic testing and quality-of-life assessment of adults with primary malignant brain tumors. Int J Radiat Oncol Biol Phys 2003; 55: 992-9.

37. Biegler KA, Chaoul MA, Cohen L. Cancer, cognitive impairment and mediation. Acta Oncol 2009; 48: 18-26.

38. Sterckx W, Coolbrandt A, Dierckx de Casterle B, Van den Heede K, Decruyenaere $\mathrm{M}$, et al. The impact of high-grade glioma on everyday life: A systematic review from patient's and caregiver's perspective. Eur J Oncl Nurs 2013; 17: 107-17. 